\title{
Sistema Computacional de Apoio à Preparação para o ENEM: uma Investigação da Acessibilidade para Surdos
}

\author{
Maíra Codo Canal $^{1}$, Laura Sánchez García ${ }^{1}$, Roberto Pereira ${ }^{1}$ \\ ${ }^{1}$ Departamento de Informática - Universidade Federal do Paraná (UFPR) \\ Caixa Postal 19.081 - 81.531-980 - Curitiba - PR - Brasil \\ mairapdn@gmail.com, \{laura, rpereira\}@inf.ufpr.br
}

\begin{abstract}
The adoption of Computer-Based Assessment (CBA) has been increasingly practiced in order to evaluate the processes involved in learning. However, for this type of evaluation produce benefits to all students, it is necessary to take into account the accessibility of such resources. This article presents an investigation of the accessibility of the types of questions used in the CBA by the Geekie Games and Redação Nota 1000 environments in the context of deaf students. This research results indicate accessibility problems in Geekie Games and Redação Nota 1000 environments. According to the identified problems, deaf students face interaction difficulties in using this type of assessment in those environments, and are even prevented from using these environments as the other students.
\end{abstract}

Resumo. A adoção da Avaliação Baseada em Computador (CBA, do inglês Computer - Based Assessment) tem sido cada vez mais praticada com o intuito de avaliar os processos envolvidos na aprendizagem. No entanto, para que este tipo de avaliação possa trazer benefícios a todos os estudantes, é necessário levar em consideração a acessibilidade de tais recursos. Este artigo apresenta uma investigação da acessibilidade dos tipos de questões usadas na CBA dos ambientes Geekie Games e Redação Nota 1000 no contexto dos estudantes surdos. Resultados dessa investigação apontam problemas de acessibilidade nos ambientes Geekie Games e Redação Nota 1000. De acordo com os problemas identificados, os estudantes surdos podem encontrar dificuldades de interação no uso desse tipo de avaliação nos ambientes, e até mesmo ficar impedidos de utilizar esses ambientes como os demais estudantes.

\section{Introdução}

A avaliação é reconhecida como um elemento-chave nos processos de ensino e aprendizagem devido ao seu potencial de promover capacitação, direção, motivação dos estudantes e, ainda, fornecer critérios necessários para medir o progresso da aprendizagem [Sim et al. 2004]. Se bem projetada, a CBA tem o potencial de promover um avanço educacional, pois a automatização da avaliação pode facilitar os procedimentos de planejamento, entrega e análise da avaliação quando comparada à avaliação com o uso de lápis e papel [Croft et al. 2001]. A CBA pode ser composta de diferentes tipos de questões, como: múltipla escolha, dissertação, lacuna, entre outros.

Estudos, como os de Luephattanasuk et al. (2001) e Beech (2011), relatam que o uso da CBA pode trazer benefícios aos professores e estudantes, mas que para isso é necessário levar em consideração a acessibilidade de tais recursos, de modo que todos 
os estudantes possam se beneficiar desse tipo de avaliação, incluindo aqueles com deficiência. Investigar a acessibilidade de recursos de CBA é, portanto, essencial para se favorecer o acesso participativo e universal do cidadão ao conhecimento - desafio \#4 da Sociedade Brasileira de Computação [Baranauskas e Souza 2006], evitando a criação de barreiras tecnológicas que resultem na exclusão de pessoas com deficiência.

No contexto brasileiro, estudantes surdos relatam dificuldades ao realizar o Exame Nacional do Ensino Médio (ENEM), que é realizado com o uso de lápis e papel e com a interação via conteúdos textuais. Esses estudantes também reivindicam o uso do computador no processo de avaliação do ENEM por causa de benefícios possibilitados pela utilização de vídeos em Libras para a tradução dos conteúdos textuais quantas vezes forem necessárias para compreensão. "O aluno poderia fazer a prova em seu tempo, retomando o vídeo quando necessário", disse um estudante surdo ${ }^{1}$. Especialistas pedagogos afirmam que a reivindicação do uso do computador no processo de avaliação do ENEM realmente pode trazer benefícios aos surdos.

O Ministério da Educação tem incentivado o uso do ambiente Geekie Games ${ }^{2}$ para a preparação dos estudantes para o ENEM. Este ambiente disponibiliza bancos de questões e possui parceria com a plataforma Redação Nota $1000^{3}$, na qual os estudantes podem se preparar para a redação do ENEM. Em 2017, está previsto que os estudantes surdos poderão realizar a prova com o uso de vídeo em Libras via computador ${ }^{4}$. Nesse sentido, há uma demanda de que esses ambientes sejam acessíveis no contexto dos surdos para que eles possam fazer o uso da CBA como os demais estudantes.

Este trabalho apresenta uma avaliação de acessibilidade dos tipos de questões usadas na CBA em Ambientes Virtuais de Aprendizagem (AVAs) tendo como foco estudantes surdos. Essa avaliação foi realizada por especialistas em Tecnologia da Informação com base no conjunto de recomendações de acessibilidade para surdos proposto por Canal e García (2015). A avaliação considerou os tipos de questões disponibilizadas pelo ambiente Geekie Games (e.g., múltipla escolha) e pelo Redação Nota 1000 (e.g., dissertação). Os resultados apontam problemas de acessibilidade relacionados ao uso de vídeos, imagens, textos, personalização pelo usuário e colaboração entre os estudantes. Os problemas identificados sugerem que estudantes surdos podem encontrar dificuldades de interação no uso desses ambientes e até mesmo serem impedidos de utilizar esses ambientes como os demais estudantes. Tal fato se configura como um fator de exclusão causado pela tecnologia que, ao excluir pessoas surdas prejudica sua autonomia, dificulta seu processo de aprendizagem e, consequentemente, as desfavorece na disputa por vagas em instituições de ensino públicas, aprofundando a desigualdade.

\footnotetext{
${ }^{1}$ Uol. http://educacao.uol.com.br/noticias/2014/11/03/estudantes-surdos-tem-dificuldade-para-sepreparar-para-o-enem.htm

2 Geekie Games. https://geekiegames.geekie.com.br

3 Redação Nota 1000. https://www.redacaonota1000.com.br/

${ }^{4}$ InfoEnem. https://www.infoenem.com.br/enem-2017-candidatos-surdos-terao-direito-video-com-provaem-libras/
} 
VI Congresso Brasileiro de Informática na Educação (CBIE 2017)

Anais do XXVIII Simpósio Brasileiro de Informática na Educação (SBIE 2017)

\section{Revisão bibliográfica}

Estudos relatam que há aceitação de professores e alunos com relação ao uso de CBA e, ainda, que o desempenho dos alunos pode ser igual ao até mesmo superior quando comparado à avaliação com uso de lápis e papel. No entanto, professores se demonstram preocupados com o uso desse tipo de avaliação pelos estudantes com deficiência e indicam que a CBA deve conter recursos acessíveis para a inclusão desses estudantes [Thompson 2000; Siozos et al. 2009].

Thompson et al. (2002) analisaram as implicações do uso de CBA para alunos com deficiência e concluíram que o uso desse tipo de avaliação tem o potencial de melhorar o processo e os resultados da avaliação dos alunos devido à possibilidade de incorporar certas acomodações, como por exemplo o uso de leitor de telas. No entanto, o uso de CBA pelos estudantes com deficiência pode ser desvantajoso se suas necessidades de acesso não forem consideradas desde o início de todo o processo de desenvolvimento [Russell et al. 2009].

No contexto dos surdos, Cawthon et al. (2011) realizaram um estudo em salas de aula com estudantes surdos no qual os conteúdos das avaliações eram transmitidos a esses estudantes pelo uso do computador por meio de vídeos em língua de sinais e as questões eram respondidas pelos estudantes de maneira escrita em papel. Resultados desse estudo indicam que os estudantes surdos obtiveram bom desempenho nos testes devido à compreensão do conteúdo transmitido nas avaliações pelos vídeos em língua de sinais.

Alguns trabalhos da literatura, (e.g., Canal e García (2014;2015)) investigaram a acessibilidade dos tipos de questões usadas na CBA oferecida pelo ambiente Moodle 2.8 no contexto dos estudantes surdos. Resultados desses trabalhos indicam problemas de interação que precisam ser tratados para que esse tipo de avaliação também possa trazer benefícios aos surdos.

No Brasil, o Ministério da Educação (MEC) tem recomendado o Geekie Games para que os estudantes se preparem para o $\mathrm{ENEM}^{5}$. Este ambiente conta com mais de três milhões de estudantes inscritos que já obtiveram, em média, uma melhora de $30 \%$ no seu desempenho no $\mathrm{ENEM}^{6}$. O Geekie Games é um ambiente online que oferece conteúdos gratuitos para apoiar a preparação para o ENEM. Além de conteúdos apresentados em textos e vídeo-aulas para estudo, o ambiente apresenta a possibilidade de o estudante realizar os testes assim como no ENEM. O diferencial desse ambiente é o fato de identificar as dificuldades dos estudantes conforme a realização dos testes e de sugerir os conteúdos necessários para estudo. Dessa maneira, o ambiente pode possibilitar a melhora do estudante nos temas abordados nos quais o estudante demonstrou dificuldade de acordo com sua pontuação obtida no processo de avaliação.

O Geekie Games tem parceria com o ambiente online Redação Nota 1000, que provê recursos para que estudantes possam elaborar e enviar suas redações que são avaliadas por especialistas segundo os critérios do Instituto Nacional de Pesquisas e Estudos Educacionais (INEP), órgão responsável pelo ENEM ${ }^{7}$. Com o uso do Redação

\footnotetext{
5 http://www.brasil.gov.br/educacao/2014/08/saiba-como-estudar-para-o-enem-pela-internet

6 http://www.geekie.com.br/sou-aluno/

${ }^{7}$ http://educacao.estadao.com.br/noticias/geral,plataforma-ajuda-, 1745450
} 
Nota 1000, os estudantes podem elaborar redações e receber o seu texto corrigido, com sua nota, um relato dos seus pontos fortes e fracos, e sugestões para reescrever sua redação $^{8}$. O estímulo do MEC para o uso do Geekie Games e do Redação Nota 1000 pelos estudantes brasileiros torna mandatório investigar a acessibilidade desses ambientes para que eles possam ser utilizados sem barreiras pelos estudantes com deficiência.

\section{Método}

O método adotado para a realização deste trabalho consiste no uso de um conjunto de recomendações de acessibilidade para surdos dos tipos de questões usadas na CBA em AVAs propostas por Canal e García (2015). Com o uso dessas recomendações, quatro especialistas em TI realizaram uma avaliação de acessibilidade dos tipos de questões usadas na CBA disponíveis nos ambientes Geekie Games e Redação Nota 1000. A avaliação dos tipos de questões foi realizada com relação à sua aplicação na elaboração das respostas pelos estudantes.

\subsection{Recomendações}

O conjunto de recomendações de acessibilidade usadas neste trabalho é formado por 34 recomendações organizadas em nove categorias (i.e., "Vídeo em Língua de Sinais", "Apresentação Alternativa para Áudio", "Apresentação Alternativa para Texto Escrito", "Apresentação Alternativa para Conteúdo Visual", "Conteúdo", "Navegação", "Dicionários e Glossários de Termos", "Colaboração entre os Estudantes" e "Feedback") que são aplicadas de acordo com 6 tipos de questões (i.e., "múltipla escolha", "verdadeiro/falso", "associação", "lacuna", "resposta curta" e "dissertação") e sua aplicação em perguntas e respostas. Para cada recomendação há três perfis (i.e., professor, estudante e desenvolvedor) com sugestões para auxiliar na sua aplicação.O objetivo dessas recomendações é prover suportes computacionais acessíveis dos tipos de questões usadas na CBA em AVAs para surdos.

\subsection{Sujeitos e procedimentos do estudo}

Quatro especialistas em TI participaram da atividade de avaliação: dois doutorandos e um mestrando em ciência da computação da Universidade Federal do Paraná e um docente da mesma instituição. Desses especialistas, três são pesquisadores da área de Interação Humano-Computador (IHC) e realizam pesquisas de acessibilidade para surdos. Todos os quatro especialistas já estavam familiarizados com o conteúdo e a aplicação dessas recomendações.

Como os avaliadores não conheciam os ambientes, antes do início da atividade o pesquisador apresentou os sistemas com o objetivo de familiarizá-los. Além disso, o pesquisador, também apresentou aos participantes os objetivos da avaliação e um site com as recomendações, orientando os participantes sobre a realização da avaliação. Todos os participantes assinaram um TCLE. Os participantes foram orientados a realizar a avaliação dos tipos de questões na visão do estudante. Cada tipo de questão foi avaliado por dois participantes individualmente.

\footnotetext{
${ }^{8}$ http://info.geekie.com.br/parceria-garante-correcoes-de-redacao-a-usuarios-da-geekie/
} 
Para realizar a avaliação, dois participantes acessaram um simulado de 2015 no ambiente Geekie Games, em que tiveram acesso a um banco de questões do tipo múltipla escolha, e dois especialistas acessaram o Redação Nota 1000, onde questões do tipo dissertação estavam disponíveis para a elaboração de redações. Durante a atividade, os participantes deveriam acessar as recomendações e indicar os resultados no formulário de anotações.

O pesquisador ficou presente para observar a interação dos participantes com os ambientes e realizou anotações sobre os comentários dos participantes. Durante a avaliação, o participante era livre para fazer questionamentos e comentários sobre os ambientes e as recomendações, e ainda interagir da maneira que julgasse necessário.

\section{Resultados}

Com base nas recomendações de acessibilidade de Canal e García (2015), os especialistas identificaram problemas de acessibilidade para surdos no Geekie Games e no Redação Nota 1000. Os resultados encontrados são apresentados nesta seção. Alguns dos problemas identificados são apresentados de acordo com a categoria à qual pertence à recomendação que não foi atendida. Algumas das recomendações que foram contempladas pelos ambientes também são destacadas na seção de resultados.

\subsection{Dados coletados}

Os dois especialistas que avaliaram o tipo de questão múltipla escolha no Geekie Games identificaram que algumas recomendações não se aplicavam à avaliação, como as recomendações da categoria "Apresentação Alternativa para Áudio", devido ao fato de o ambiente não apresentar nenhum tipo de conteúdo de áudio nas questões. Os outros dois especialistas que avaliaram o Redação Notal 1000 relataram que também não utilizaram as recomendações da categoria "Apresentação Alternativa para Áudio" no processo de avaliação, pois o ambiente não apresenta conteúdo na forma de áudio nas questões. Os avaliadores também relataram que a recomendação da categoria "Feedback" não foi aplicada na avaliação devido ao fato de o acesso ao feedback da resposta da questão dissertativa não ser imediato.

Categoria: Vídeo de língua de sinais. Alguns dos problemas encontrados pelos especialistas que avaliaram os dois ambientes foram relacionados à recomendação "1. A qualidade do vídeo deve permitir que sejam captados detalhes sobre os movimentos das mãos, olhos e boca". Todos os participantes identificaram que os ambientes não disponibilizam vídeos em línguas de sinais sobre os conteúdos que são apresentados na questão para o estudante. Os especialistas também identificaram que não é possível fazer o uso de vídeos em língua de sinais para responder o tipo de questão dissertação. Portanto, como o ambiente não faz uso de vídeo em língua de sinais nos tipos de questões, todos os especialistas concluíram que a recomendação de número 1 não foi atendida.

Outro problema encontrado pelos especialistas que avaliaram os tipos de questões é que os ambientes não disponibilizam vídeo em língua de sinais referente ao conteúdo da questão apresentado em texto para compreensão da pergunta pelo estudante. Assim, os participantes da avaliação concluíram que os ambientes não contemplam a recomendação " 2 . Prover vídeo em língua de sinais a todo texto escrito". Outro problema encontrado pelos especialistas em TI referente à recomendação 2 é a impossibilidade de gravar vídeos em língua de sinais ou, ainda, de carregar vídeos em 
VI Congresso Brasileiro de Informática na Educação (CBIE 2017)

Anais do XXVIII Simpósio Brasileiro de Informática na Educação (SBIE 2017)

adição à resposta em texto no tipo de questão dissertativa que provê ao estudante um campo para preenchimento da resposta.

Categoria: Apresentação alternativa para texto escrito. Os especialistas identificaram que ao responder o tipo de questão múltipla escolha, o Geekie Games disponibiliza questões que são elaboradas com o uso de textos juntamente com gráficos e imagens. No entanto, também identificaram que o ambiente apresenta algumas questões que são elaboradas somente com o uso de conteúdos textuais, conforme apresenta a Figura 1. Dessa maneira, o ambiente não atende a recomendação "9. Prover recursos visuais aos conteúdos apresentados em texto (ex. complementar texto com apresentações gráficas ou visuais)".



Figura 1. Exemplo de questão múltipla escolha do Geekie Games composta unicamente de texto.

Os outros dois avaliadores também identificaram que algumas questões do tipo dissertação apresentam o uso de imagens além de conteúdos textuais. No entanto, também identificaram que algumas questões só utilizam conteúdos textuais em seu enunciado. Ainda, os especialistas também concluíram que não é possível adicionar imagens na resposta do tipo de questão dissertação. Portanto, o ambiente não atende a recomendação 9 , pois o estudante não pode adicionar imagens ao responder esse tipo de questão.

Categoria: Apresentação alternativa para conteúdo visual. Os avaliadores identificaram que a recomendação "13. Usar a Língua de sinais (vídeo ou avatar) para explicar o conteúdo do banner ou figura" não é contemplada pelo Geekie Games nem pelo Redação Notal 1000. Todos os especialistas identificaram que as imagens utilizadas nos enunciados das questões dos ambientes possuem apenas conteúdos explicativos em textos. Ainda com relação ao tipo de questão dissertação, os especialistas identificaram que os estudantes não podem gravar vídeos em língua de sinais para explicar o conteúdo de banner ou figura para responder as questões no campo de resposta do ambiente Redação Nota 1000.

Categoria: Conteúdo. Os dois especialistas que avaliaram o Geekie Games identificaram que o ambiente não disponibiliza links para que o estudante possa ter acesso a informações adicionais referentes ao conteúdo da questão do tipo múltipla 
VI Congresso Brasileiro de Informática na Educação (CBIE 2017)

Anais do XXVIII Simpósio Brasileiro de Informática na Educação (SBIE 2017)

escolha, não atendendo a recomendação "16. Incluir links adicionais para reunir informações detalhadas". Por outro lado, os dois especialistas que avaliaram o Redação Nota 1000 identificaram que o ambiente contempla a recomendação 16, pois o estudante pode acessar conteúdos adicionais sobre o enunciado da questão. A Figura 2 apresenta os links que são oferecidos pelo ambiente ao estudante para que possa ter acesso a informações adicionais sobre o tema da dissertação a ser elaborada.

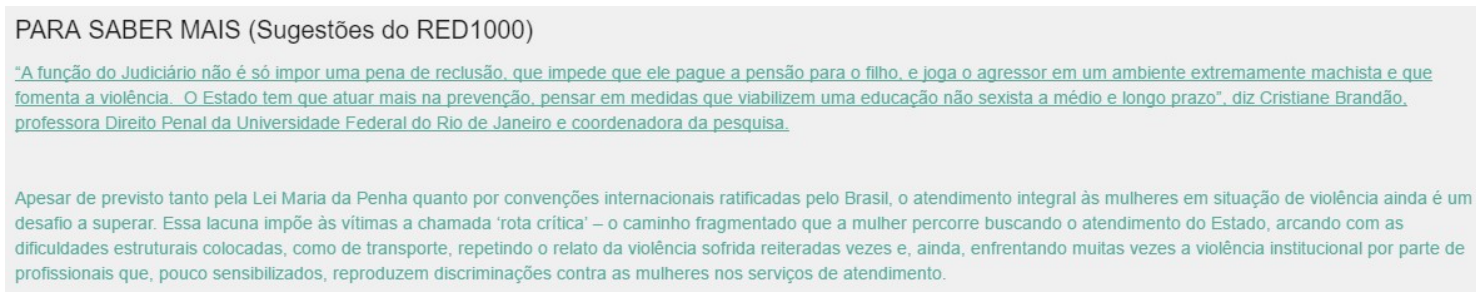

Figura 2. Links que contém informações adicionais sobre o tema da redação disponibilizados ao estudante.

Categoria: Dicionários e glossários de termos. Todos os especialistas identificaram que os estudantes não possuem acesso a dicionários e glossários de termos ao responder tanto o tipo de questão múltipla escolha no Geekie Games quanto o tipo de questão dissertação no Redação Nota 100. Assim, os especialistas concluíram que as recomendações "21. Oferecer um dicionário e glossário de termos" e "22. Dicionários de tradução de texto podem ser oferecidos para palavras individuais ou frases" não são atendidas por ambos os ambientes.

Categoria: Navegação. Os especialistas identificaram que os ambientes não disponibilizam opções para que o estudante tenha acesso ao conteúdo da questão de acordo com sua preferência, não atendendo a recomendação " 25 . Fornecer opções para que os usuários possam receber documentos de acordo com suas preferências (por exemplo, a linguagem, tipo de conteúdo, etc). No caso do usuário surdo, que ele possa escolher entre o Português ou a Língua de Sinais.

Ainda com relação à navegação, na avaliação do Geekie Games, os avaliadores identificaram que a recomendação "26. Capacidade de o estudante poder repetir as instruções quantas vezes ele desejar" foi contemplada, pois após responder uma questão, o estudante pode avançar para a próxima questão ou voltar à questão anterior já respondida e alterar sua resposta. Com relação à avaliação no Redação Nota 1000, os especialistas também afirmaram que a recomendação é contemplada, pois o estudante pode repetir as instruções da questão quantas vezes desejar.

Os especialistas também identificaram que nenhum dos ambientes oferece a opção de configuração de cores. Dessa maneira, o estudante não pode escolher a cor de texto e a cor de fundo da tela conforme suas preferências. Assim, os avaliadores afirmam que os ambientes não contemplam a recomendação "29. Configuração de cores".

Categoria: Colaboração entre os estudantes. Os avaliadores identificaram que os dois ambientes não atendem a recomendação "33. Prover recursos para aprendizagem em grupos via vídeo conferência. Considerar a possibilidade de se propor situações onde seja possível realizar atividades em grupo ou em dupla" pois não oferecem recursos para a aprendizagem colaborativa. Nesse sentido, como se trata da preparação para o ENEM, a interação entre os estudantes via Geekie Games ou Redação Nota 1000 poderia trazer 
benefícios devido à troca de conhecimentos e à aprendizagem colaborativa apoiadas pelas ferramentas no momento da execução das questões.

Categoria: Feedback. Outro problema de acessibilidade encontrado pelos avaliadores foi com relação à recomendação "34. Prover feedback em relação às atividades do estudante usando mídia (ex. texto, imagem, vídeo) e língua (ex. português, língua de sinais) de acordo com a preferência do estudante". Segundo esta recomendação, o estudante deve poder escolher a maneira que o feedback é apresentado. Os dois especialistas concluíram que o Geekie Games apresenta somente o feedback em forma de texto, não permitindo a visualização do feedback com o uso de vídeo e/ou imagem. Desta forma, o estudante surdo não pode receber o feedback da maneira que julgar melhor para o seu entendimento, podendo afetar a sua compreensão da correção do exercício. A Figura 3 apresenta um exemplo de feedback retirado do Geekie Games.



Figura 3. Exemplo de feedback retirado do ambiente Geekie Games.

\section{Discussão}

As avaliações realizadas pelos especialistas possibilitaram a obtenção de informações relevantes sobre a acessibilidade dos tipos de questões dos ambientes Geekie Games e Redação Nota 1000, demonstrando que ambos os sistemas possuem limitações de interface e interação para o público surdo. A inclusão de estudantes surdos passa pela solução dos problemas de acessibilidade identificados neste trabalho de forma a viabilizar o uso desse tipo de avaliação para todos os estudantes, incluindo aqueles com deficiência.

Os dois especialistas que avaliaram o tipo de questão múltipla escolha encontraram os mesmos problemas para o tipo de questão no Geekie Games. Assim como os dois especialistas encontraram os mesmos problemas para o tipo de questão dissertação no Redação Nota 1000. Também, os dois especialistas que avaliaram o tipo de questão dissertação encontraram as mesmas recomendações que o Redação Nota 1000 contempla. O fato também se repetiu com os avaliadores do Geekie Games. Dessa maneira, o uso das recomendações serviu de apoio para a investigação dos tipos de questões usadas em CBA oferecidas pelo Geekie Games e pelo Redação Nota 1000.

Alguns problemas de acessibilidade identificados no ambiente Geekie Games se repetiram na identificação de problemas no Redação Nota 1000, como por exemplo, o 
não oferecimento de vídeos em língua de sinais aos conteúdos das questões, a falta de recursos para apoiar a colaboração entre os estudantes, a falta de recursos para a personalização pelo usuário no conteúdo da questão e sobre a configuração de cores.

Os problemas de acessibilidade encontrados na avaliação pelos especialistas têm relação com os problemas já identificados por Canal e García $(2014 ; 2015)$ na avaliação dos tipos de questões do ambiente Moodle 2.8. Por exemplo, (1) a impossibilidade de adição de outras mídias, que não texto às alternativas de resposta disponíveis ao estudante no tipo de questão dissertação e (2) a impossibilidade do estudante selecionar o tipo de feedback no tipo de questão múltipla escolha. Outro problema que se repetiu no Moodle e nos ambientes avaliados neste trabalho foi com relação à opção para que o estudante escolha a maneira que deseja acessar o conteúdo das questões (português ou língua de sinais). Esses resultados sugerem que os mesmos problemas prevalecem em diferentes plataformas que possuem propósitos equivalentes de uso, como o Moodle, o Geekie Games e o Redação Nota 1000.

As recomendações que foram atendidas nos tipos de questão do Geekie Games e do Redação Nota 1000, também foram atendidas pelo Moodle ("16" e "26"). Nesse sentido, seria relevante levantar questões sobre a proposição e a verificação de soluções para os problemas encontrados e, assim, impulsionar a inclusão dos estudantes surdos.

\section{Conclusões e trabalhos futuros}

Este trabalho apresenta uma avaliação de acessibilidade dos tipos de questões usadas em CBA oferecidas pelos ambientes Geekie Games e Redação Nota 1000 no contexto dos estudantes surdos. Para tanto, utilizou-se um conjunto de recomendações de acessibilidade para surdos dos tipos de questões usadas na CBA em AVAs. A avaliação foi realizada por especialistas em TI que utilizaram as recomendações para avaliar os tipos de questões múltipla escolha e dissertação com relação à compreensão do enunciado e da elaboração da resposta no papel do estudante nos ambientes.

Os resultados da avaliação apontam diversos problemas de acessibilidade nos tipos de questões múltipla escolha e dissertação dos ambientes no contexto dos estudantes surdos que envolvem o uso de vídeos em língua de sinais, imagens, textos, personalização pelo usuário e colaboração entre os estudantes. Os resultados da avaliação também indicam que o uso do conjunto de recomendações na avaliação apoiou os avaliadores e possibilitou a identificação de problemas de acessibilidade.

No contexto estudado, os estudantes concorrerão pelo acesso ao ensino superior público. Se um sistema computacional disponibilizado para apoiar a capacitação desses estudantes possui problemas de acessibilidade, ele pode acabar por ampliar a desigualdade de oportunidades e assim favorece um grupo de pessoas ao mesmo tempo em que exclui outro. Especialmente em contextos relacionados à educação e ao exercício da cidadania, a falta de acessibilidade tem um impacto difícil de ser mensurado e identificado, gerando consequências sociais que não podem ser revertidas ou corrigidas com a atualização do sistema técnico.

Trabalhos futuros incluem avaliar os ambientes com o uso das recomendações utilizadas neste trabalho sob a perspectiva da elaboração dos enunciados das questões pelo (a) professor (a) e realizar a avaliação dos tipos de questões dos ambientes também com estudantes surdos. Finalmente, é relevante estender a investigação para se 
VI Congresso Brasileiro de Informática na Educação (CBIE 2017)

Anais do XXVIII Simpósio Brasileiro de Informática na Educação (SBIE 2017)

considerar outros tipos de deficiência sob a luz do Design Universal e assim oferecer propostas de soluções para os problemas encontrados nos ambientes estudados.

\section{Referências}

Baranauskas, M.C.C. e Souza, C.S. (2006). "Desafio $\mathrm{n}^{\circ}$ 4: Acesso participativo e universal do cidadão brasileiro ao conhecimento". In: Computação Brasil, ano VII, $\mathrm{n}^{\mathrm{o}} 23, \mathrm{p} .7$.

Beech, M. (2011). "Computer-based testing accommodations for students with disabilities". Technical report. Ph.D. Learning Systems Institute. Florida State University. Bureau of Exceptional Education and Student Services Florida Department of Education.

Canal, M.C. e García, L.S. (2015). "Recomendações de acessibilidade para surdos dos tipos de questões usadas na avaliação baseada em computador em ambientes virtuais de aprendizagem". In: XXVI Simpósio Brasileiro de Informática na Educação (SBIE), p. 812-821.

Canal, M.C. e García, L.S. (2014). "Research on Accessibility of Question Modalities Used in Computer-Based Assessment (CBA) for Deaf Education". Universal Access in Human-Computer Interaction. Universal Access to Information and Knowledge. Lecture Notes in Computer Science, Volume 8514, pp 265-276.

Cawthon, S.W., Winton S.M., Garberoglio, C.L. e Gobble, M.E. (2011). "The effects of american sign language as an assessment accommodation for students who are deaf or hard of hearing". Journal of Deaf Studies and Deaf Education, 16(2):198-211.

Croft, A.C., Danson, M., Dawson, B.R., Ward, J.P. (2001). "Experiences of using computer assisted assessment in engineering mathematics." Computers \& Education, 27:53-66.

Debevc, M., Povalej, M., Verli£, M. e Stjepanovi£, Z. (2007). "Exploring usability and accessibility of an e-learning system for improving computer literacy". New Trends in ICT \& Accessibility, 119-124.

Luephattanasuk, N., Suchato, A. e Punyabukkana, P. (2011). "Accessible QTI presentation for web-based elearning". In Proceedings of the International CrossDisciplinary Conference on Web Accessibility. ACM, Article 26.

Russell, M., Hoffmann, T. e Higgins, J. (2009). "Meeting the needs of all students: A universal design approach to computer-based testing". Journal of Online Education, $5(4)$.

Russell, M., Kavanaugh, M., Masters, J., Higgins, J. e Hoffmann, T. (2009). "Computer-based signing accommodations: Comparing a recorded human with an avatar.” Journal of Applied Testing Technology, 10(3).

Sim, G., Holifield, P. e Brown, M. (2004). "Implementation of computer assisted assessment: Lessons from the literature". Association for Learning Technology Journal, 12(3), 215-229.

Thompson, S.J., Thurlow, M.L., Quenemoen, R.F. e Lehr, C.A. (2002). "Access to computer-based testing for students with disabilities". (synthesis report 45) Technical report. University of Minnesota, National Center on Educational Outcomes, Minneapolis. 